\title{
Low-Damage and High-Rate Sputtering of Silicon Surfaces by Ethanol Cluster Ion Beam
}

\section{$\operatorname{AUTHOR}(\mathrm{S})$ :}

Ryuto, Hiromichi; Sugiyama, Kazumichi; Ozaki, Ryosuke; Takaoka, Gikan H.

\section{CITATION:}

Ryuto, Hiromichi ...[et al]. Low-Damage and High-Rate Sputtering of Silicon Surfaces by Ethanol Cluster Ion Beam. Applied Physics Express 2009, 2: 016504.

\section{ISSUE DATE:}

2009-02

URL:

http://hdl.handle.net/2433/89641

\section{RIGHT:}

c 2009 The Japan Society of Applied Physics; This is not the published version. Please cite only the published version.; この論文は出版社版で ありません。引用の際には出版社版をご確認ご利用ください。 


\section{Low-Damage and High-Rate Sputtering of Silicon Surfaces by Ethanol Cluster Ion Beam}

Hiromichi Ryuto*`, Kazumichi Sugiyama, Ryosuke Ozaki, and Gikan H. Takaoka

Photonics and Electronics Science and Engineering Center, Kyoto University, Kyoto 615-8510, Japan

To realize the high-rate and low-damage sputtering of a Si surface, the effect of irradiating an ethanol cluster ion beam on a Si surface was investigated. The sputtering depths in Si substrates induced by the ethanol cluster ion beam irradiation were larger than those in $\mathrm{SiO}_{2}$ substrates, which was due to a chemical sputtering effect. The lattice disorder and the surface roughness of the Si substrates decreased with increasing retarding voltage.

${ }^{*}$ E-mail address: ryuto@ @uee.kyoto-u.ac.jp

${ }^{\dagger}$ Formerly, Hiromichi Akiyoshi 
The density of integrated circuits has been continuously increasing since their first appearance around $1960 .{ }^{1)}$ In the nanotechnology era, the surface properties of materials have become critical for the efficiency of devices because of their rapidly increasing density; thus, the requirements for material processing methods have become exacting. ${ }^{2,3)}$ For example, ion-beam etching and implantation are common methods in semiconductor manufacturing. However, the damage caused by the ion-beam irradiation is a serious drawback to the requirement of lower-damage processing for higher-density devices. On the other hand, the cluster ion beam method realizes the irradiation of electromagnetically controlled ion beams that are more than 100 times heavier than the heaviest atomic ion beam, a ${ }^{238} \mathrm{U}$ beam. Various cluster ion beams such as metal, ${ }^{4,5)}$ fullerene, ${ }^{6)}$ silicon, ${ }^{7)}$ 'gas', ${ }^{8)}$ and 'liquid, ${ }^{9)}$ cluster ion beams have attracted much interest. The unique features of the interaction between cluster ion beams and solid materials ${ }^{8)}$ are mainly attributable to the high-density energy deposition and the lowenergy irradiation effect. Since the kinetic energy of a molecule in a cluster is equal to the total kinetic energy divided by the cluster size (the number of molecules that comprise a cluster), low-energy ion beams can be realized using cluster ion beams at relatively high acceleration voltages. Therefore, the radiation damage caused by cluster ion beams is much smaller than that caused by monomer ion beams. A liquid cluster ion beam also has unique properties that are induced by the characteristic structure as well as the chemical properties of the molecules that comprise the cluster. The interactions of liquid cluster ions with solid surfaces have been investigated to clarify the characteristics of liquid clusters as a new type of liquid materials. Their characteristics are expected to be much different from those of bulk-state materials used in conventional wet processes. Furthermore, it is of much importance to clarify the effects of irradiating liquid cluster ions, and this study is expected to open up a new field of materials science. In this article, a method of achieving low-damage and high-rate sputtering using an ethanol cluster ion beam is presented.

Figure 1 shows a schematic view of the liquid-cluster ion-beam apparatus. The apparatus consists of three vacuum chambers: a source chamber, an ionization chamber, and a target chamber. The chambers are separately evacuated using vacuum pumps that meet the gas load of the chambers. For example, the source chamber is evacuated using a Roots pump and the ionization chamber is evacuated using a diffusion pump. Ethanol is filled in a liquid container, which is placed in the source chamber. Typically about $150 \mathrm{~cm}^{3}$ of ethanol is used for approximately $2 \mathrm{~h}$ of irradiation. The ethanol is heated using a heater attached to the outer wall of the container, and is maintained at the temperature corresponding to the vapor pressure at which the clusters with the desired size distribution is produced. The source temperature was $109^{\circ} \mathrm{C}$, which corresponds to a vapor pressure of $3 \mathrm{~atm}$. The typical variation of the temperature is approximately $\pm 1^{\circ} \mathrm{C}$, which corresponds to a $\pm 10 \mathrm{kPa}$ variation of the ethanol vapor pressure. Vaporized ethanol is ejected to the source chamber through a Laval nozzle whose nozzle diameter is $0.1 \mathrm{~mm}$. The expanding vapor is supersaturated, and clusters are produced. ${ }^{10)}$ The core portion of the flow is selected by a skimmer and transported to the ionization chamber through a chamber for differential pumping. The diameter of the skimmer opening is $1.3 \mathrm{~mm}$. The skimmer is 
used to avoid the disintegration of clusters caused by shock waves. The Mach-disk position has sometimes been estimated using the empirical formula for sonic nozzles, ${ }^{11)}$ but the formula cannot be used to predict when a Laval or conical nozzle is used. ${ }^{12,13)}$ In the ionization chamber, the neutral cluster beam is ionized by the bombardment of electrons emitted from the loop of a tungsten filament. The current and voltage of the ionization electrons are typically $200 \mathrm{~V}$ and $0.2 \mathrm{~A}$, respectively. The ionized beam is extracted by an extraction voltage applied to an extraction electrode. The typical extraction voltages are from 3 to $9 \mathrm{kV}$. The lower part of the cluster size distribution was eliminated using a retarding voltage $(\mathrm{Vr})$ applied to a retarding electrode. This method is based on the phenomenon that the velocity of the nozzle flow is highly uniform, typically within $10 \%{ }^{10,14)}$ The kinetic energy of ethanol clusters calculated on the basis of this phenomenon is $0.29 \mathrm{eV} /$ molecule. The size-separated cluster ion beam is accelerated by an acceleration voltage (Va) typically from 3 to $9 \mathrm{kV}$ applied to an acceleration electrode. The divergence of the accelerated cluster ion beam is suppressed using an Einzel lens, and the horizontal position of the beam is fine-tuned using an electrostatic deflector. A Faraday cup with an electron suppressor typically at $-315 \mathrm{~V}$ and a sample holder attached to a linear motion feedthrough are inserted at the beam position. The vertical intensity distribution of the cluster ion beam is measured by moving the Faraday cup, and the sample is placed at the peak of the distribution. The variation of the beam intensity over time was typically within $10 \%$. The samples irradiated in this work were $\mathrm{Si}(111)$ and $\mathrm{SiO}_{2}$ substrates at room temperature. The effect of the charge-up in the $\mathrm{SiO}_{2}$ substrate was expected to be small because the mass-to-charge ratio of the ethanol cluster ions is extremely large. The sputtering depths of the samples were measured using a step profiler. The number of disordered atoms in the Si samples was measured by the Rutherford backscattering (RBS) method using $\alpha$ particles at $2 \mathrm{MeV}$. The surface roughness was measured by an atomic force microscope (AFM).

The cluster size distributions were measured by the time-of-flight (TOF) method. ${ }^{15)}$ The peak positions of the distributions were located between 1300 to 1800 when retarding voltages of 27 to $82 \mathrm{~V}$ were applied, respectively, and the distributions shifted to the larger side in general as the retarding voltage was increased. Figure 2 shows the retarding voltage dependences of the sputtering depths of (a) $\mathrm{Si}$ and (b) $\mathrm{SiO}_{2}$ substrates irradiated with ethanol cluster ion beams. The $\mathrm{SiO}_{2}$ samples were irradiated with a 5 times larger dose than the Si samples to improve the accuracy of the depth profile measurement. The sputtering depths in the Si samples at the retarding voltage of $27 \mathrm{~V}$ were approximately 6 times larger than those in the $\mathrm{SiO}_{2}$ samples considering the linear dependence of the sputtering depth on the dose. The maximum sputtering yield in the Si sample irradiated at the acceleration voltage of $9 \mathrm{kV}$ was 360 atoms/ion, which is more than 200 times larger than that for an Ar monomer ion beam at $9 \mathrm{keV}$. The large sputtering yields in the Si samples are possibly due to the chemical sputtering effect. ${ }^{16)}$ The sputtering depths of both the $\mathrm{Si}$ and $\mathrm{SiO}_{2}$ substrates irradiated at higher acceleration voltages were larger than those obtained at lower acceleration voltages. In the case of the acceleration voltages of 6 and $9 \mathrm{kV}$, the sputtering depths of the Si substrates initially increased as the retarding 
voltage increased, then decreased when the retarding voltage further increased to above the peak values. The initial increase may be explained by the range of the cluster ions in Si and the volatility of the chemical products generated from the reaction between ethanol and Si. In the present case, the cluster size increases with increasing retarding voltage, and the kinetic energy per incident ethanol molecule decreases. Therefore, the range of the ethanol cluster ions in the Si may be larger when the retarding voltage is smaller. If the ethanol cluster ions were deeply implanted in the Si samples, the evaporation of the chemical products would be blocked. Therefore, when the retarding voltage is smaller than the peak value, a lower retarding voltage may result in a smaller sputtering depth. The decrease in the sputtering depth when the retarding voltage is further increased may be explained by the following mechanism. The kinetic energy per molecule of the larger part of the cluster size distribution may be too small to induce sputtering, and the ratio of this part increases as the retarding voltage is increased. The sputtering depth for the acceleration voltage of $3 \mathrm{kV}$ monotonically decreased as the retarding voltage increased. This is possibly because the range is already smaller than the depth where the evaporation of the chemical products is prevented, even when the retarding voltage is less than $30 \mathrm{~V}$. On the other hand, the sputtering depth in the $\mathrm{SiO}_{2}$ samples monotonically decreased with increasing retarding voltage. The reason for this tendency may be explained by assuming that the interaction between an ethanol cluster and $\mathrm{SiO}_{2}$ substrate is dominated by the physical sputtering and by considering the decrease in the kinetic energy per molecule with the increase in retarding voltage.

Figure 3 shows the retarding voltage dependences of the number of disordered atoms in the $\mathrm{Si}$ substrates measured by the RBS of $\alpha$ particles at $2 \mathrm{MeV}$. The number of disordered atoms (ND) is recognized as a measure of radiation damage. ${ }^{17)}$ The sample irradiated at the acceleration voltage of 3 $\mathrm{kV}$ and a retarding voltage smaller than $54 \mathrm{~V}$ had a finite ND value, which approached 0 as the retarding voltage increased. This phenomenon is thought to be because the ratio of ethanol molecules that have sufficiently large kinetic energies to produce lattice disorders decreased as the retarding voltage was increased. However, in the cases of 6 and $9 \mathrm{kV}$, all the data points are located at approximately the same values. So far, the whole peak near the surface in the channeling spectra was assumed to originate from the lattice disorders, but it may be possible that some parts of the peak originate from silicon dioxide or other products formed by the interaction between ethanol molecules and silicon atoms. The phenomena observed in the cases of 6 and $9 \mathrm{kV}$ can possibly be explained by this assumption.

Figure 4 shows the retarding voltage dependences of the surface roughness of (a) $\mathrm{Si}$ and (b) $\mathrm{SiO}_{2}$ substrates. The surface roughness of the Si substrates irradiated with the ethanol cluster ion beam was larger than that of the unirradiated surface. However, the surface roughness decreased with increasing retarding voltage, and the surface roughness was smaller than $1 \mathrm{~nm}$ when the retarding voltage was larger than approximately $50 \mathrm{~V}$. Moreover, in the case of $\mathrm{SiO}_{2}$ samples irradiated with the ethanol cluster ion beam at an acceleration voltage of $3 \mathrm{kV}$, the surface roughness decreased to that of the unirradiated sample as the retarding voltage was increased. This phenomenon may be explained by the enhancement of the lateral sputtering effect caused by the increase in the cluster size with increasing 
retarding voltage.

In summary, the effect of irradiating an ethanol cluster ion beam on a Si surface was investigated to examine the possibility of low-damage as well as high-rate sputtering. The sputtering depths of Si substrates were deeper than those of $\mathrm{SiO}_{2}$ substrates at the energy range from 3 to $9 \mathrm{keV}$. On the other hand, in the case of the $3 \mathrm{kV}$ acceleration voltage, the number of disordered $\mathrm{Si}$ atoms decreased to that of the unirradiated sample with increasing retarding voltage. A surface roughness of less than 1 $\mathrm{nm}$ was realized at retarding voltages larger than approximately $50 \mathrm{~V}$. Therefore, the low-damage and high-rate sputtering of Si substrates can be realized using an ethanol cluster ion beam by adjusting the retarding and acceleration voltages.

\section{Acknowledgement}

The RBS measurements were performed at the Quantum Science and Engineering Center, Kyoto University. 


\section{References}

1) G. E. Moore: Electronics 38 (1965) 114.

2) J. E. Greene: MRS Bulletin 26 (2001) 777.

3) J. W. Faul and D. Henke: Nucl. Instrum. Meth. B 237 (2005) 228.

4) I. Yamada and G. H. Takaoka: Jpn. J. Appl. Phys. 32 (1993) 2121.

5) A. Herrmann, S. Leutwyler, E. Schumacher, and L. Wöste: Helv. Chim. Acta 61 (1978) 453.

6) H. Shen, C. Brink, P. Hvelplund, S. Shiryaev, P. X. Shi, and J. A. Davies: Nucl. Instrum. Meth. B 129 (1997) 203.

7) Y. Iwata, M. Kishida, M. Muto, S. Yu, T. Sawada, A. Fukuda, T. Takiya, A. Komura, and K. Nakajima: Chem. Phys. Lett. 358 (2002) 36.

8) I. Yamada: Nucl. Instrum. Meth. B 148 (1999) 1.

9) G. H. Takaoka, H. Noguchi, T. Yamamoto, and T. Seki: Jpn. J. Appl. Phys. 42 (2003) L1032.

10) O. F. Hagena and W. Obert: J. Chem. Phys. 56 (1972) 1793.

11) H. Ashkenas and F. Sherman: in Rarefied Gas Dynamics, ed. J. H. de Leeuw (Academic Press, New York, 1966) vol. II, p. 84.

12) A. Benidar, R. Georges, R. Le Doucen, J. Boissoles, S. Hamon, A. Canosa, and B. R. Rowe: J. Mol. Spectrosc. 199 (2000) 92.

13) A. Broc, S. De Benedictis, G. Dilecce, M. Vigliotti, R. G. Sharafutdinov, and P. A. Skovorodko: J. Fluid Mech. 500 (2004) 211.

14) O. F. Hagena and K. Varma: Rev. Sci. Instrum. 39 (1968) 47.

15) G. H. Takaoka, H. Noguchi, K. Nakayama, and M. Kawashita: Surf. Sci. Nanotech. 4 (2006) 473.

16) G. H. Takaoka, M. Kawashita, and T. Okada: Rev. Sci. Instrum. 79 (2008) 02 C503.

17) W.-K. Chu, J. W. Mayer, and M.-A. Nicolet: Backscattering Spectrometry (Academic Press, New York, 1978). 
Figure Captions:

Fig. 1: Schematic view of liquid-cluster ion-beam apparatus.

Fig. 2: Retarding voltage dependences of sputtering depth of (a) $\mathrm{Si}$ and (b) $\mathrm{SiO}_{2}$ substrates irradiated with ethanol cluster ion beams at acceleration voltages of 3 (circles), 6 (triangles), and $9 \mathrm{kV}$ (squares).

Fig. 3: Retarding voltage dependences of number of disordered atoms in Si substrates irradiated with ethanol cluster ion beams at acceleration voltages of 3 (circles), 6 (triangles), and $9 \mathrm{kV}$ (squares), measured by the RBS method. The error bars represent only the statistical error of the RBS measurement.

Fig. 4: Retarding voltage dependences of surface roughness of (a) $\mathrm{Si}$ and (b) $\mathrm{SiO}_{2}$ substrates irradiated with ethanol cluster ion beams at acceleration voltages of 3 (circles), 6 (triangles), and $9 \mathrm{kV}$ (squares). 


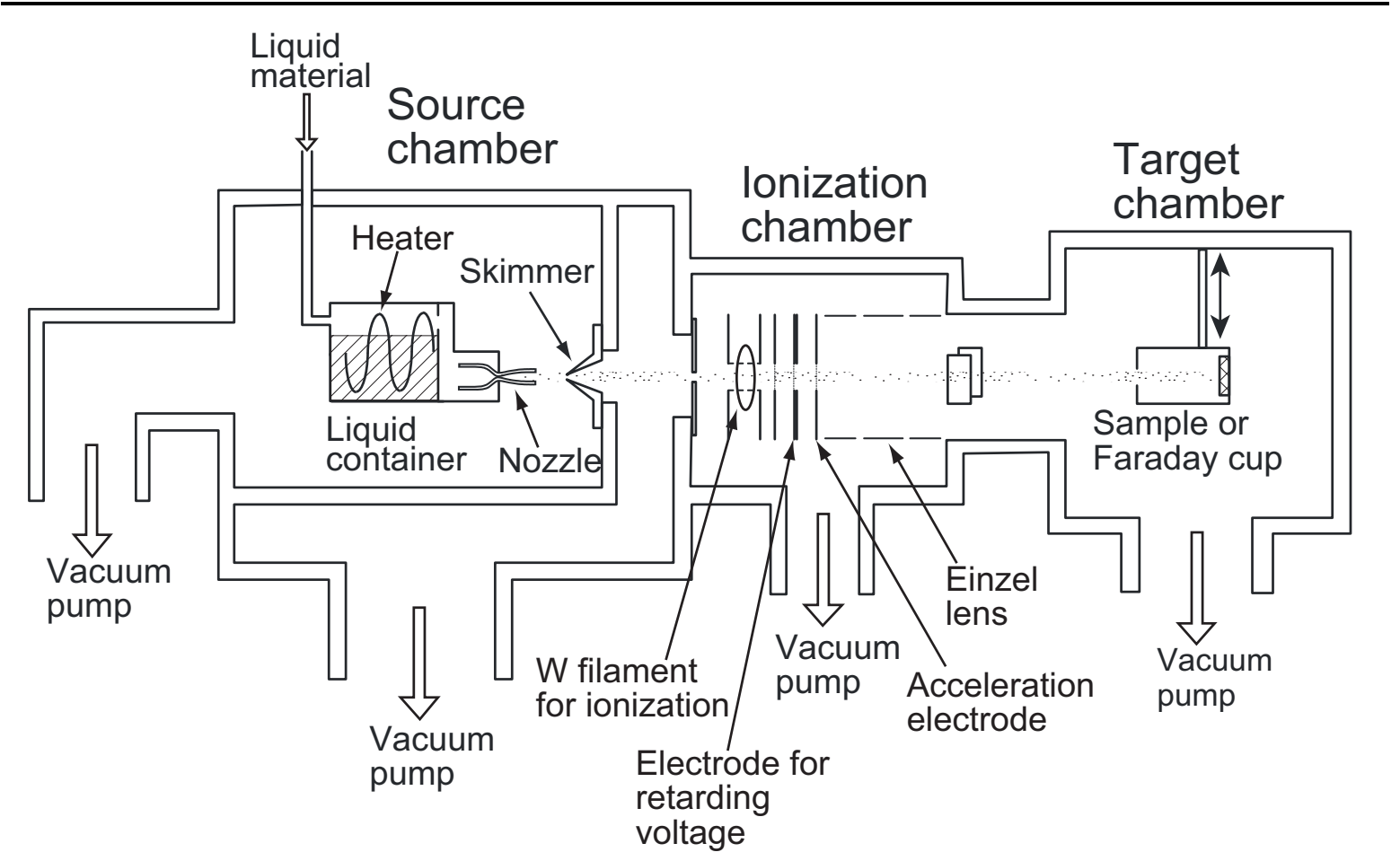

Fig. 1. 


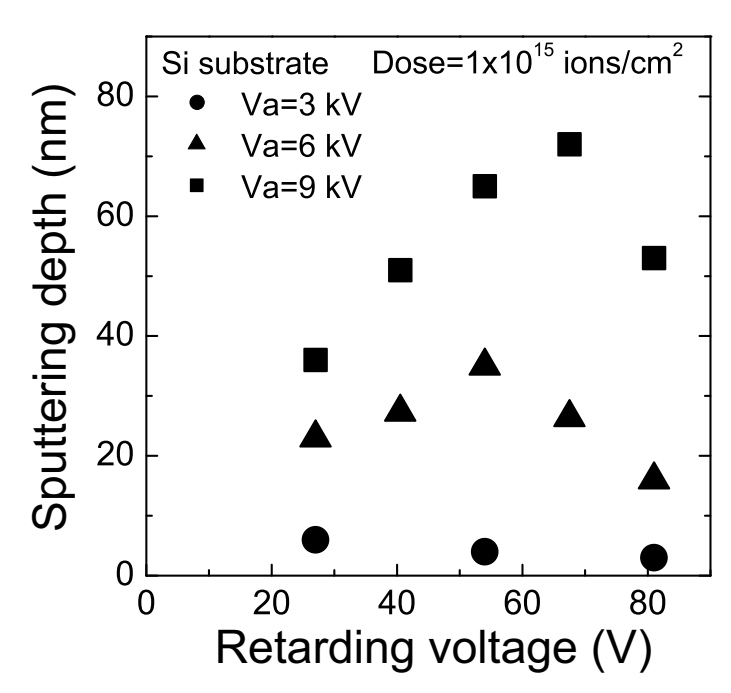

(a)

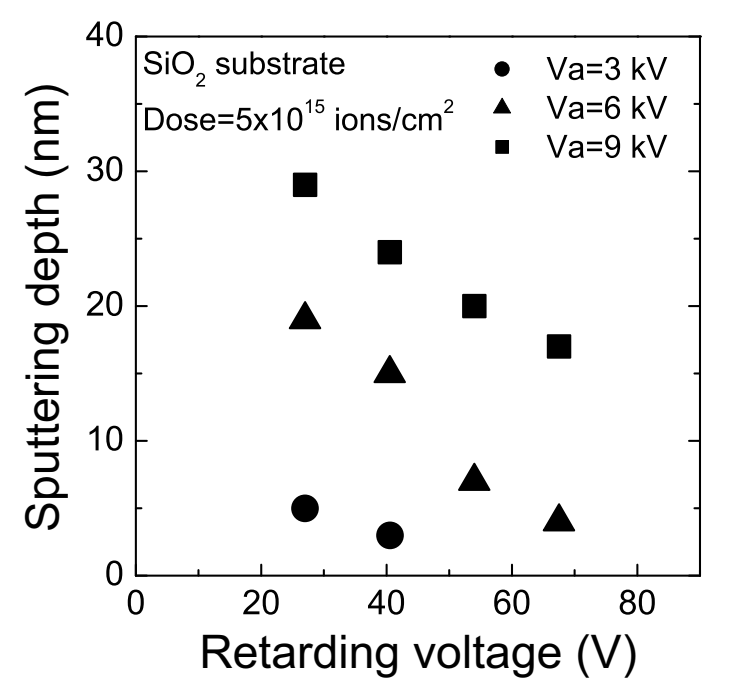

(b)

Fig. 2. 


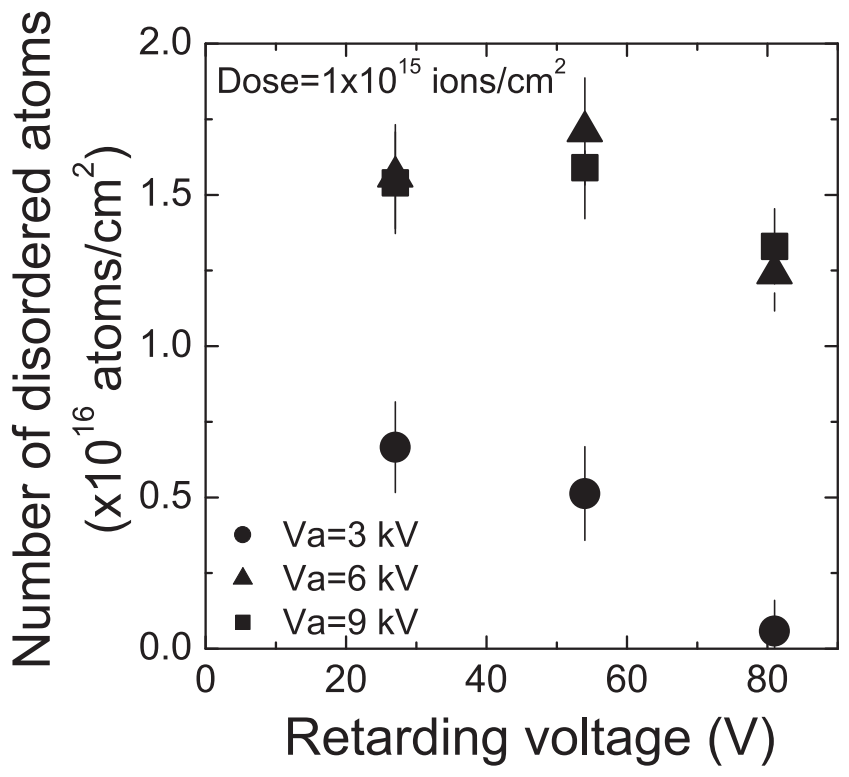

Fig. 3. 


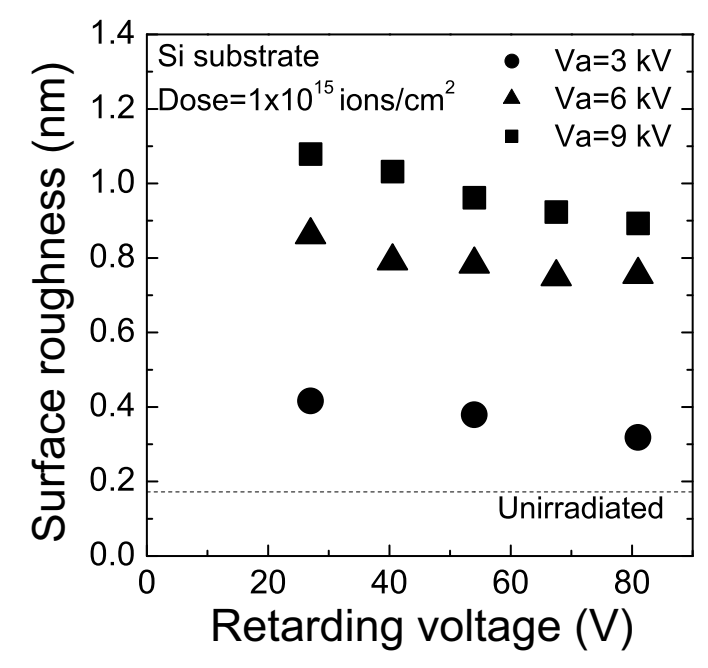

(a)

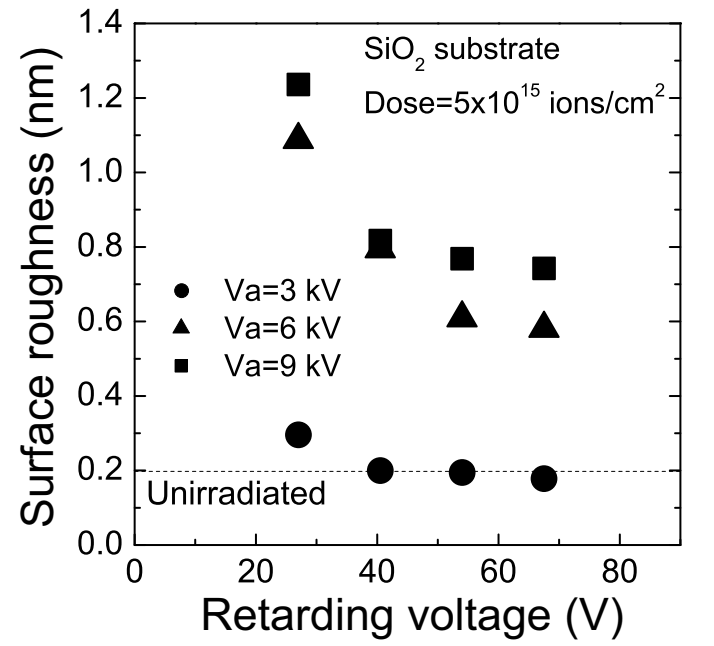

(b)

Fig. 4. 